\title{
Heat-induced Proteome Changes in Tomato Leaves
}

\author{
Suping Zhou', Roger J. Sauvé, Zong Liu, Sasikiran Reddy, and Sarabjit Bhatti \\ Department of Agricultural Sciences, School of Agriculture and Consumer Sciences, Tennessee State \\ University, 3500 John A. Merritt Boulevard, Nashville, TN 37209 \\ Simon D. Hucko, Yang Yong, Tara Fish, and Theodore W. Thannhauser \\ Plant, Soil and Nutrition Research Unit, USDA-ARS, Tower Road, Ithaca, NY 14853-2901
}

\begin{abstract}
Additional Index words. Solanum lycopersicum, heat stress, proteomics, photosynthesis, methionine and SAM biosynthesis, glycolate shunt, Rubisco activase, transketolase

Abstract. Three tomato (Solanum lycopersicum) cultivars [Walter LA3465 (heat-tolerant), Edkawi LA 2711 (unknown heat tolerance, salt-tolerant), and LA1310 (cherry tomato)] were compared for changes in leaf proteomes after heat treatment. Seedlings with four fully expanded leaves were subjected to heat treatment of $39 / 25^{\circ} \mathrm{C}$ at a $16: 8 \mathrm{~h}$ light-dark cycle for 7 days. Leaves were collected at $1200 \mathrm{HR}, 4 \mathrm{~h}$ after the light cycle started. For 'Walter' LA3465, heat-suppressed proteins were geranylgeranyl reductase, ferredoxin-NADP $(+)$ reductase, Rubisco activase, transketolase, phosphoglycerate kinase precursor, fructose-bisphosphate aldolase, glyoxisomal malate dehydrogenase, catalase, S-adenosyl-L-homocysteine hydrolase, and methionine synthase. Two enzymes were induced, cytosolic NADP-malic enzyme and superoxide dismutase. For 'Edkawi' LA2711, nine enzymes were suppressed: ferredoxinNADP $(+)$ reductase, Rubisco activase, S-adenosylmethionine synthetase, methioine synthase, glyoxisomal malate dehydrogenase, enolase, flavonol synthase, M1 family peptidase, and dihydrolipoamide dehydrogenase. Heat-induced proteins were cyclophilin, fructose-1,6-bisphosphate aldolase, transketolase, phosphoglycolate phosphatase, ATPase, photosystem II oxygen-evolving complex 23, and NAD-dependent epimerase/dehydratase. For cherry tomato LA1310, heat-suppressed proteins were aminotransferase, S-adenosyl-L-homocysteine hydrolase, L-ascorbate peroxidase, lactoylglutathione lyase, and Rubisco activase. Heat-induced enzymes were glyoxisomal malate dehydrogenase, phosphoribulokinasee, and ATP synthase. This research resulted in the identification of proteins that were induced/repressed in all tomato cultivars evaluated (e.g., Rubisco activase, methionine synthase, adenosyl-Lhomocysteine hydrolase, and others) and those differentially expressed (e.g., transketolase).
\end{abstract}

Temperature is a key factor determining optimal growth and productivity of plants. In recent decades, many parts of North America have been experiencing an increase in the number of unusually hot days and nights. Continued global warming is likely to result in an increase in frequency and intensity of heat waves and drought (U.S. Global Change Research Program, 2008). Leaves function as the primary manufacturer of many metabolites used during plant growth. Both the integrity of the machinery and functionality of enzymes associated with photosynthetic activity are sensitive to heat stress (Berry and Björkman, 1980; Murakami et al., 2000). Significant inhibition of photosynthesis occurs at temperatures only a few degrees above the optimum, resulting in a considerable loss of potential productivity.

Tomato is one of the most important vegetable species and cash crops in the world. Daytime temperatures consistently above $32^{\circ} \mathrm{C}$ and evening temperatures that stay above $24^{\circ} \mathrm{C}$ are considered excessive and are detrimental to tomato plant growth and fruit development. Tomato leaves exposed to prolonged heat stress conditions experience starch depletion as a result of enhanced hydrolysis and reduced biosynthesis activities (Dinar et al., 1983). Short-term heat stress affects

Received for publication 7 Jan. 2011. Accepted for publication 7 Mar. 2011. This project is supported by the Agriculture and Food Research Initiative competitive grant no. 2010-65114-20405 from the USDA National Institute of Food, and Agriculture, 1890 Capacity Building Program, and Evans-Allen Research Funds.

We thank Drs. Nick Gawel and Daifeng Hui at Tennessee State University for critical review of the manuscript.

${ }^{1}$ Corresponding author. E-mail: zsuping@tnstate.edu. pollination, resulting in unfertilized embryos and aborted fruits (Berry and Rafique-Ud-Din, 1988).

Previous research has demonstrated that there is a strong correlation between heat stress and fruit yield in tomato (Berry and Rafique-Ud-Din, 1988). Membrane thermostability and heat-induced increase in chlorophyll a:b ratio and decrease in chlorophyll:carotenoids ratio are directly associated with the level of thermotolerance in tomato cultivars (Camejo et al., 2005; Saeed et al., 2007). Individual genes encoding for heat stress transcription factors (Chan-Schaminet et al., 2009; Scharf et al., 1998; Schultheiss et al., 1996), heat shock and chaperonin proteins (Port et al., 2004), and other functional proteins [e.g., kinases, reactive oxygen species scavengers, enzymes associated with sugar metabolism (Frank et al., 2009; Link et al., 2002)] play key roles in modulating thermotolerance in tomato.

Damage resulting from heat stress is present in multiple forms such as oxidative burst (Camejo et al., 2006), metabolic toxicity, membrane disorganization, inhibition of photosynthesis, and altered nutrient acquisition (Ismail and Hall, 1999; Karim et al., 1999; Wahid et al., 2007). Tolerant plants have a higher capacity to maintain homeostasis under stress by the activation of stress perception and signaling pathways, antioxidant capacity, gene expression regulation pathways, and alteration of metabolic cycles (Bohnert et al., 2006, and references therein). As a result of the complexity of plant response to heat stress, very few tolerant cultivars have been produced using traditional breeding protocols. Genetic transformation techniques have been of little use as a result of limited knowledge and availability of genes with known effects on plant heat-stress tolerance (Foolad, 2005; Wahid et al., 
2007). In this project, heat-induced changes in the whole proteomes of tomato leaves were identified using a proteomics approach. The objective of this research was to determine candidate genes and pathways that should be investigated when breeding tolerant tomato cultivars.

\section{Materials and Methods}

Plant growth and heat treatment. Three tomato cultivars \{Walter LA3465 [heat-tolerant (Swift, 2008)], Edkawi LA2711 [unknown heat tolerance, salt-tolerant], and LA1310 [cherry tomato]\} were compared. Seed stocks were obtained from the C.M. Rick Tomato Genetics Resource Center at the University of California, Davis. Seeds were propagated in a greenhouse at Tennessee State University (Nashville). For this project, tomato seeds were germinated in seed cubes (SmithersOasis, Kent, $\mathrm{OH}$ ), and seedlings were grown to the four fully expanded mature leaf stage in a greenhouse. Before heat treatment, tomato seedlings were transferred into two illuminated incubators (Thermo Fisher Scientific, Pittsburgh, PA), which were programmed at $25^{\circ} \mathrm{C}$ and light cycle of $16 / 8 \mathrm{~h}$ (day/ night). After 1 week, the incubator for heat treatment was reprogrammed to $39 / 25{ }^{\circ} \mathrm{C}(16 / 8 \mathrm{~h}$ day/night $)$ and the other incubator for control was kept at $25^{\circ} \mathrm{C}$. Cool-white fluorescent tubes provided a photosynthesis photon flux intensity of 500 $\mu \mathrm{mol} \cdot \mathrm{m}^{-2} \cdot \mathrm{s}^{-1}$. Five plants from each cultivar were placed on each shelf, and three layers of shelves (removing the top and the third shelves) were used in an incubator. The upper three fully expanded leaves were collected at $1200 \mathrm{HR}, 4 \mathrm{~h}$ after the light cycle was initiated. Leaf tissues collected from the five plants on each shelf were pooled as one biological sample (replicate), three samples were collected for the control and treatment, and they were the three biological replicates for proteomics analysis. Immediately after detachment from the plants, the leaf samples were frozen in liquid nitrogen.

Preparation of PRotein SAMPles ANd differential TWODIMENSIONAL FLUORESCENCE GEL ELECTROPHORESIS. To extract protein, frozen leaf tissues were ground into a fine powder and mixed into acetone containing $10 \%$ trichloroacetic acid and $0.5 \%$ dithiothreitol (Sigma, St. Louis, MO). After incubation at $-20{ }^{\circ} \mathrm{C}$ overnight, protein was precipitated by centrifugation at $10,000 g_{n}$ at $4{ }^{\circ} \mathrm{C}$ for $10 \mathrm{~min}$. Pellets were washed four times with pre-chilled $100 \%$ acetone to remove all residual acid. Protein pellets were dried in a Thermo Savant SpeedVac (Thermo Fisher Scientific) at low heat.

For gel analysis, the protein powder was reswollen at room temperature in two-dimensional (2D) protein rehydration buffer consisting of $7 \mathrm{M}$ urea, $2 \mathrm{M}$ thiourea, and 4\% 3[(3cholamidopropyl) dimethylammonio]-propanesulfonic acid. Soluble proteins were separated by centrifugation at 14,000 $g_{\mathrm{n}}$ for $10 \mathrm{~min}$. The protein concentration was determined using Bradford Protein Assay Reagent (Bio-Rad, Hercules, CA).

To quantitatively compare the samples using differential 2D fluorescence gel electrophoresis (DIGE) analysis, three biological replicates were labeled with cyanine dyes $\mathrm{Cy} 3$ and Cy5 (GE Healthcare, Piscataway, NJ) according to the manufacturer's instructions. Cy-dye-labeled samples were grouped randomly during electrophoresis so that no two $\mathrm{Cy} 3$ and $\mathrm{Cy} 5$ pairs were run on duplicate gels to eliminate statistical biases (Karp and Lilley, 2007; Karp et al., 2007). A dye swap design was incorporated to control for labeling biases. A combined Cy2-labeled internal standard containing equal amounts of all the protein extractions used in the experiment was used to normalize across the multiple gels (Alban et al., 2003), which greatly reduces variation in the samples as a result of electrophoresis and loading. The dye:protein ratio for the experiments was $200 \mathrm{pmol}$ dye:50 $\mu \mathrm{g}$ total protein. The analytical gels were run using $50 \mu \mathrm{g}$ of protein from each labeled sample. A preliminary analysis on a limited number of samples was done to conduct a power analysis to facilitate the design of the largescale analysis. The pilot study demonstrated that three biological replicates were sufficient to identify differentially expressed proteins with greater than a 1.5-fold change at a statistical power of 0.85 or greater. Therefore, subsequent experiments had three biological replicates per treatment.

For running the gels, a protein sample was first subjected to isoelectric focusing (IEF) on the 24-cm Immobiline DryStrip pH 3-10 NL (GE Healthcare). At the completion of the IEF run, proteins were reduced and alkylated (Zhang et al., 2003). Strips were transferred onto $12.52 \%$ acrylamide-sodium dodecyl sulphate gels, which were prepared using $41.75 \%$ (v/v) of protogel (National Diagnostics, Atlanta, GA), and the gels $(255 \times$ $196 \times 1 \mathrm{~mm}$ ) were run on a Hoefer SE900 vertical slab gel electrophoresis unit (Hoefer, Holliston, MA) using the following protocol: $20^{\circ} \mathrm{C}$ at $20 \mathrm{~mA}$ for $30 \mathrm{~min}$ and then $50 \mathrm{~mA}$ for $12 \mathrm{~h}$ until the bromophenol blue front dye reached the bottom of the gel.

Gels were scanned on the Typhoon 9300 Variable Mode Imager (GE Healthcare) at $100 \mathrm{dpi}$ according to the manufacturer's specifications for Cy Dyes (GE Healthcare) and Colloidal Coomassie Blue (Invitrogen, Carlsbad, CA) -stained gels were visualized with the $632.8 \mathrm{~nm}$ helium-neon laser with no emission filter. The gel images were analyzed using Progenesis Samespots (Version 3.3; Nonlinear Dynamics, Newcastle Upon Tyne, UK). All images passed quality control checks for saturation and dynamic range and were cropped to adjust for positional differences in scanning. The alignment procedure was semiautomated. Fifty manual alignment seeds were added per gel ( $\approx 12$ landmark spots per quadrant) and the gels were then autoaligned and grouped according to treatment. The SameSpots default settings, for detection, background subtraction (lowest on boundary), normalization, and matching, were used. Spots (picking lists) were selected as being differentially expressed if they showed greater than a 1.5-fold change in spot density and an analysis of variance score of $P<0.05$.

For protein identification, preparative picking gels were run in which $450 \mu \mathrm{g}$ of protein was loaded. Gel preparation and electrophoresis were done following the same procedure as DIGE gels. The protein gels were stained with Colloidal Blue staining solution (Invitrogen) overnight and destained in double-distilled $\mathrm{H}_{2} \mathrm{O}$. Proteins spots were picked manually from the gels and digested in situ with trypsin (sequence-grade trypsin, $12.5 \mathrm{ng} \cdot \mathrm{mg}^{-1}$; Promega, Madison, WI) overnight. The resulting peptides were extracted from the gel pieces and concentrated with ZipTip $\mathrm{C}_{18}$ pipette tips (Millipore, Bedford, MA). An aliquot of each digest was spotted (along with matrix) onto a matrix-assisted laser desorption/ionization-mass spectrometry (MALDI-MS) target.

The samples were subjected to MALDI analysis using a 4700 Proteomics Analyzer equipped with time-of-flight (TOF)-TOF ion optics (Applied Biosystems, Framingham, MA). Before analysis, the mass spectrometer was calibrated, externally, using a six-peptide calibration standard $(4700 \mathrm{Cal}$ Mix; Applied Biosystems). Most samples were calibrated 
internally using the common trypsin autolysis products (at $\mathrm{m} / \mathrm{z}$ $842.51,1045.5642$, and 2211.1046 Da) as mass calibrants. The external calibration was used as the default if the trypsin autolysis products were not observed in the spectra of the samples. The instrument was operated in the $1 \mathrm{kV}$ positive ion reflector mode. The laser power was set to 4500 for MS and 5200 for MS/MS with collision-induced dissociation off. MS spectra were acquired across the mass range of 850 to $4000 \mathrm{Da}$. MS/MS spectra were acquired for the 10 most abundant precursor ions provided they exhibited a signal to noise ratio 25 or less. Calibration was external using the known fragments of angiotensin I (monoisotopic mass 1296.6853 Da). A maximum of 2000 laser shots was accumulated per precursor. The MS data were processed using Mascot Daemon (Matrix Science, Boston,

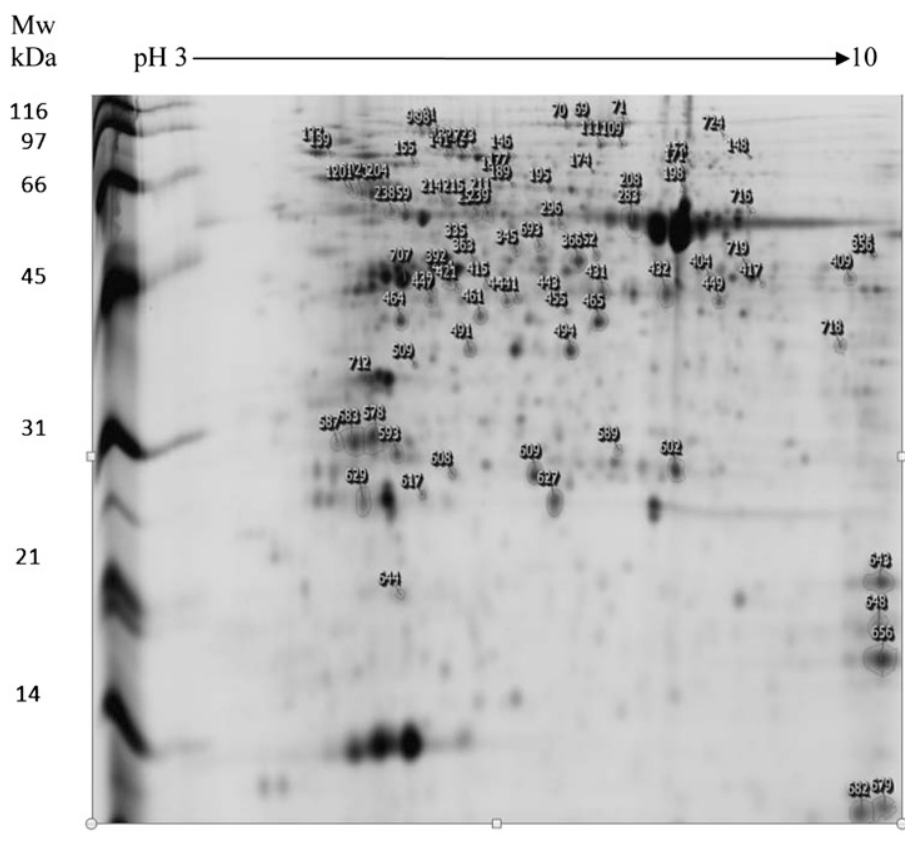

(A)

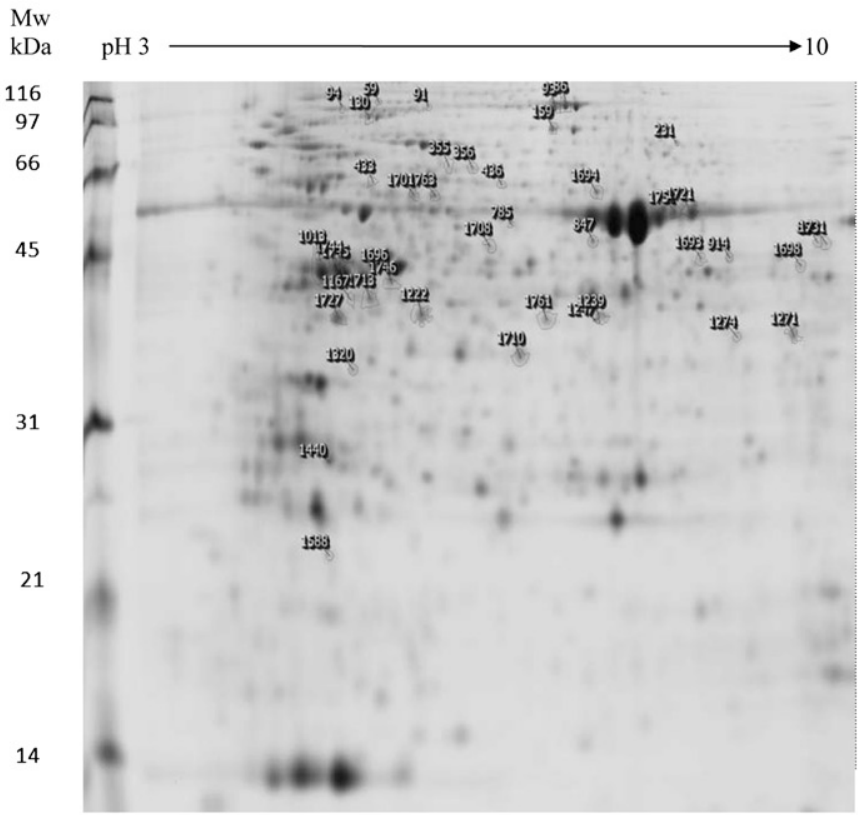

(B)

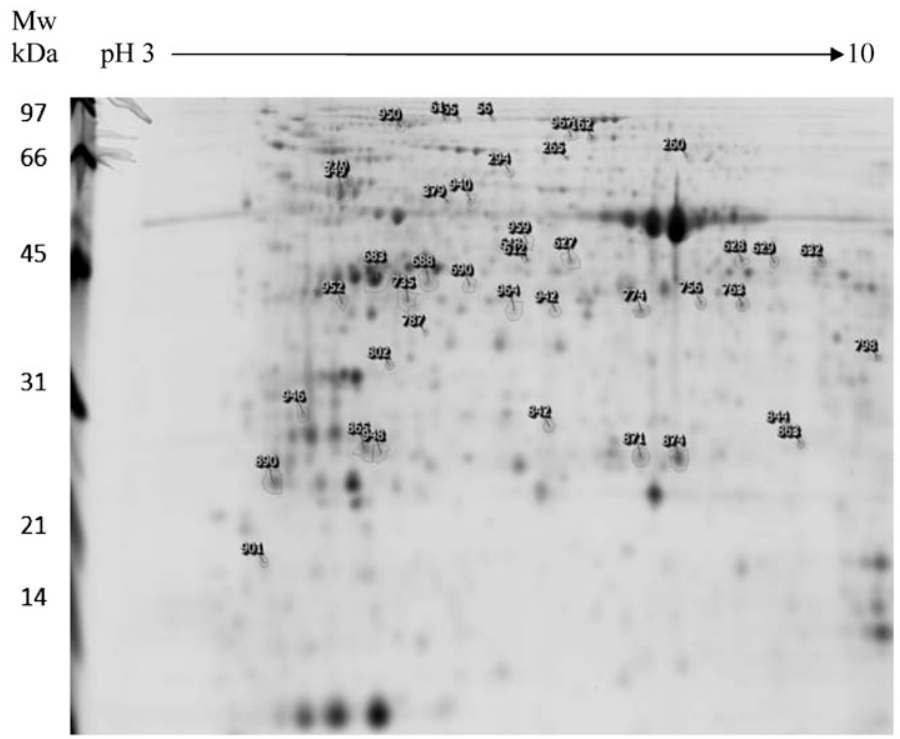

(C)

Fig. 1. Difference gel electrophoresis (DIGE) electropherogram of tomato leaf proteins after heat treatment. Heat-induced changes in leaf proteomes were identified from three tomato cultivars (A) Edkawi LA2711, (B) Walters LA3465, and (C) cherry tomato LA1310. Plants were incubated at $39^{\circ} \mathrm{C}$ (heat treatment) and $25^{\circ} \mathrm{C}$ (control) for $7 \mathrm{~d}$. Protein were labeled with cyanine dyes using the CyDye DIGE Fluors (GE Healthcare, Piscataway, NJ). Proteins from control and treated samples were labeled Cy3 and Cy5 and an internal control consisting equal amount of all protein extractions was labeled with Cy2. The Cy-dye-labeled proteins $(150 \mu \mathrm{g}$ total with $50 \mu \mathrm{g}$ from each of the three labeled samples) were focused on 24-cm Immobiline DryStrip pH 3-10 NL [GE Healthcare (shown on the x-axis)] and then separated on $12.52 \%$ acrylamide-sodium dodecyl sulphate gels. The molecular weight markers (Mw) are shown on the y-axis; they were the Cy2-labeled Broad Range Protein Molecular Weight Markers (Bio-Rad, Hercules, CA). Gels were scanned on a Typhoon 9300 Variable Mode Imager (GE Healthcare) and the images were analyzed using Progenesis Samespots program (Version 3.3; Nonlinear Dynamics, Newcastle Upon Tyne, UK). Numbered spots are protein spots that showed changes above 1.5 -fold between control and the heat treatment at below $P<0.05$ level through analysis of variance. 
MA) to submit searches to Mascot (Version 2.3; Matrix Science). The search parameters used were as follows: tryptic protease specificity, one missed cleavage allowed, $30 \mathrm{ppm}$ precursor mass tolerance, 0.5 -Da fragment ion mass tolerance with a fixed modification of cysteine carbamidomethylation, and a variable modification of methionine oxidation. Spectra were searched against an in-house tomato protein database (T.W. Thannhauser, unpublished data) created by combining 40,000 predicted proteins from the tomato UniGene build 2 release (25 Mar. 2009; National Center for Biotechnology Information, Bethesda, MD) and 9000 predicted proteins that to date had been annotated in the tomato genome release (3 May 2009; SOL Genomics Network, Ithaca, NY). Only peptides that matched with a Mascot score above the $95 \%$ confidence interval threshold $(P<0.05)$ were considered for protein identification. Only proteins containing at least one unique peptide (a sequence that had not been previously assigned to different protein) were considered.

\section{Results}

In this experiment, no visible damage was observed on the leaf surface after heat treatment. However, the number of protein spots that showed greater than 1.5 -fold $(P<0.05)$ changes between control and treated samples were different according to the cultivars. The highest number of protein spots was identified in 'Edkawi' LA2711 [86 protein spots (Fig. 1A)] followed by 'Walter' LA3465 [43 protein spots (Fig. 1B)]; cherry tomato LA1310 had the fewest number of protein spots [40 protein spots (Fig. 1C)] exhibiting significant changes after heat treatment.

Sixteen protein spots were identified in 'Walter' LA3465, of which 13 were suppressed and two induced (Table 1). Heatsuppressed proteins included two isoforms of geranylgeranyl reductase (spots 1731 and 850:-1.6- and-1.5-fold, respectively) in chlorophyll biosynthesis, ferredoxin-NADP $(+)$ reductase in the electron transport chain of PSI (spot 1710: -2.1-fold expression), Rubisco (Ribulose 1, 5-bisphosphate carboxylase/ oxygenase) activase (spots 1745 and 1013: -2.1- and -1.5-fold, respectively), and transketolase (spot 130: -1.6 -fold) related to carbon fixation, the phosphoglycerate kinase precursor (spot 1696: -2.9-fold), and fructose-bisphosphate aldolase (spot 1727: -1.5 -fold) in the glycolytic pathway, two isoforms of glyoxisomal malate dehydrogenase (spots 1222 and 1271:-1.6and -1.7 -fold, respectively) in the glycolate shunt, and Sadenosyl-L-homocysteine hydrolase (spot 1701: -2.1-fold), and methionine synthase (spot 159:-1.6-fold) in methionine, and S-adenosyl methionine (SAM) metabolism. The two heat-induced enzymes were the cytosolic NADP-malic (NADP-ME) enzyme (spot 356: 2.8-fold) and antioxidant superoxide dismutase (SOD) (spot 1588: 1.8-fold).

Table 1. Heat-induced and heat-suppressed proteins in tomato 'Walter' LA3465.

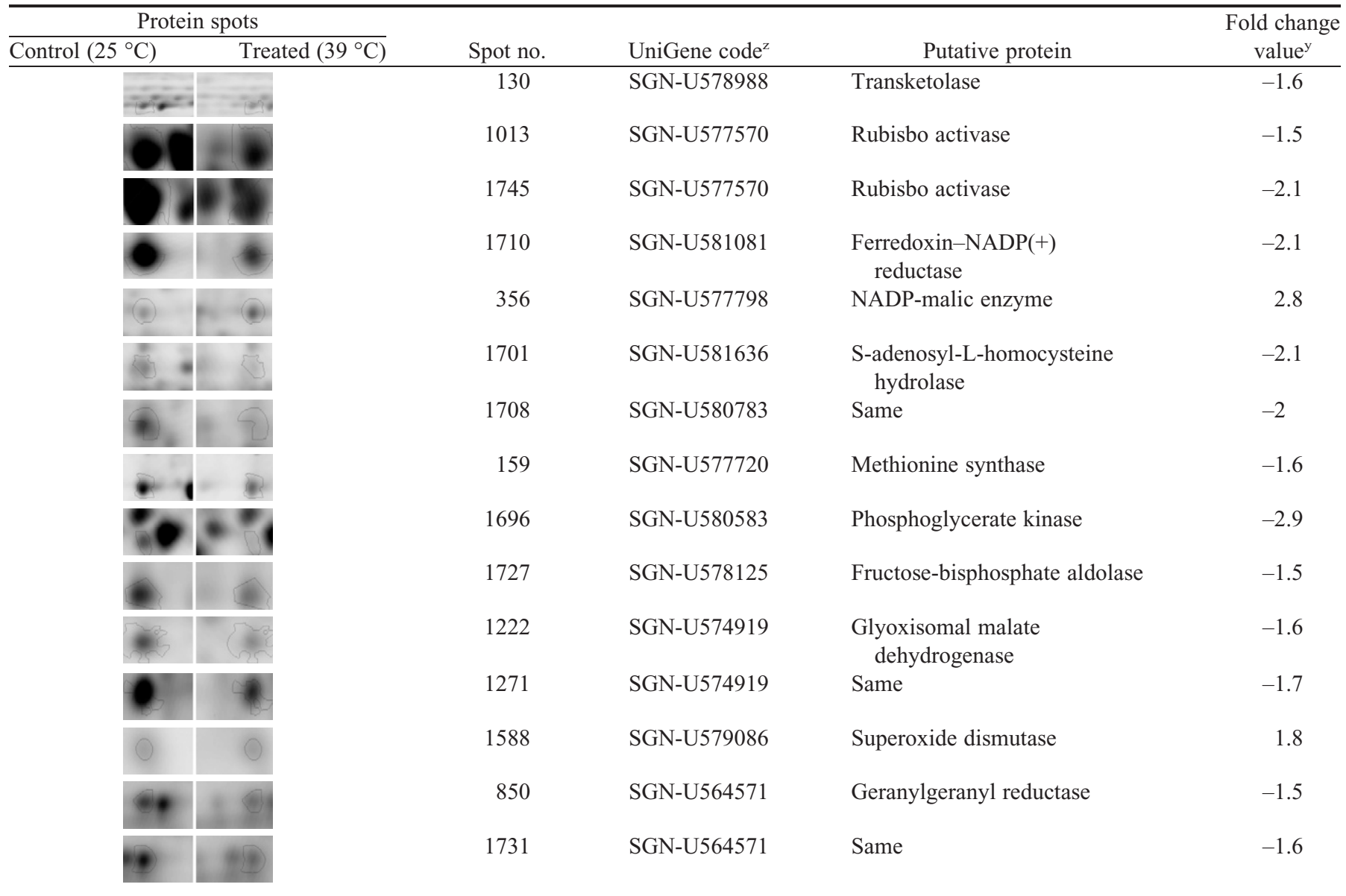

${ }^{\mathrm{z}}$ Code for each gene in the SOL Genomics Network (SGN) database.

${ }^{\mathrm{y}}$ Fold change value is the ratio of the normalized volume of the same spot in the condition of heat-treated versus control. For example, a value of 2.0 represents a twofold increase, whereas -2.0 represents a twofold decrease from treated to control conditions. 
In 'Edkawi' LA2711, 21 protein spots were identified, 11 spots were induced and 10 spots were suppressed (Table 2). Heat-suppressed proteins included ferredoxin-NADP $(+)$ reductase (spot 494: -1.9-fold), Rubisco activase isoforms (spots 707 and 392: -1.7- and -4.2-fold, respectively), S-adenosylmethionine synthetase (spot 693: -2.6-fold), and methioine synthase (spots 111 and 109: -1.8- and -3.2-fold, respectively), glyoxisomal malate dehydrogenase (spot 718 : -1.8-fold), enolase (spot 211:-2.3-fold), flavonol synthase (spot 447: -1.8-fold), two isoforms of M1 family peptidase (spots 587 and 81:-1.8- and -twofold, respectively), and dihydrolipoamide dehydrogenase (spot 208: -twofold). The heat-induced proteins were cyclophilin (CY2) protein (spot 648: 1.5-fold), fructose- 1,6-bisphosphate aldolase (spot 491: 1.8-fold), transketolase (spot 141: 2.2-fold) in the Calvin cycle, phosphoglycolate phosphatase (spot 712: 1.7-fold) in photorespiration, ATPase isoforms (spots 706, 201, and 202: 1.7-, 2.3-, and 2.6-fold, respectively), the photosystem II oxygen-evolving complex 23 (OEC23) (spot 629: twofold) of photosynthetic machinery component, and NAD-dependent epimerase/dehydratase (spot 345: 2.0-fold) in carbohydrate metabolism.

In cherry tomato LA1310 (Table 3), heat-suppressed proteins included aminotransferase (spot 627: -6.1-fold) in lysine biosynthesis; S-adenosyl-L-homocysteine hydrolase (spot 940: -3.4-fold), adenosylmethionine synthetase (spot 612: -5.8-fold) in the activated methyl cycle, antioxidant L-ascorbate peroxidase

Table 2. Heat-induced and heat-suppressed proteins in tomato 'Edkawi' LA2711.

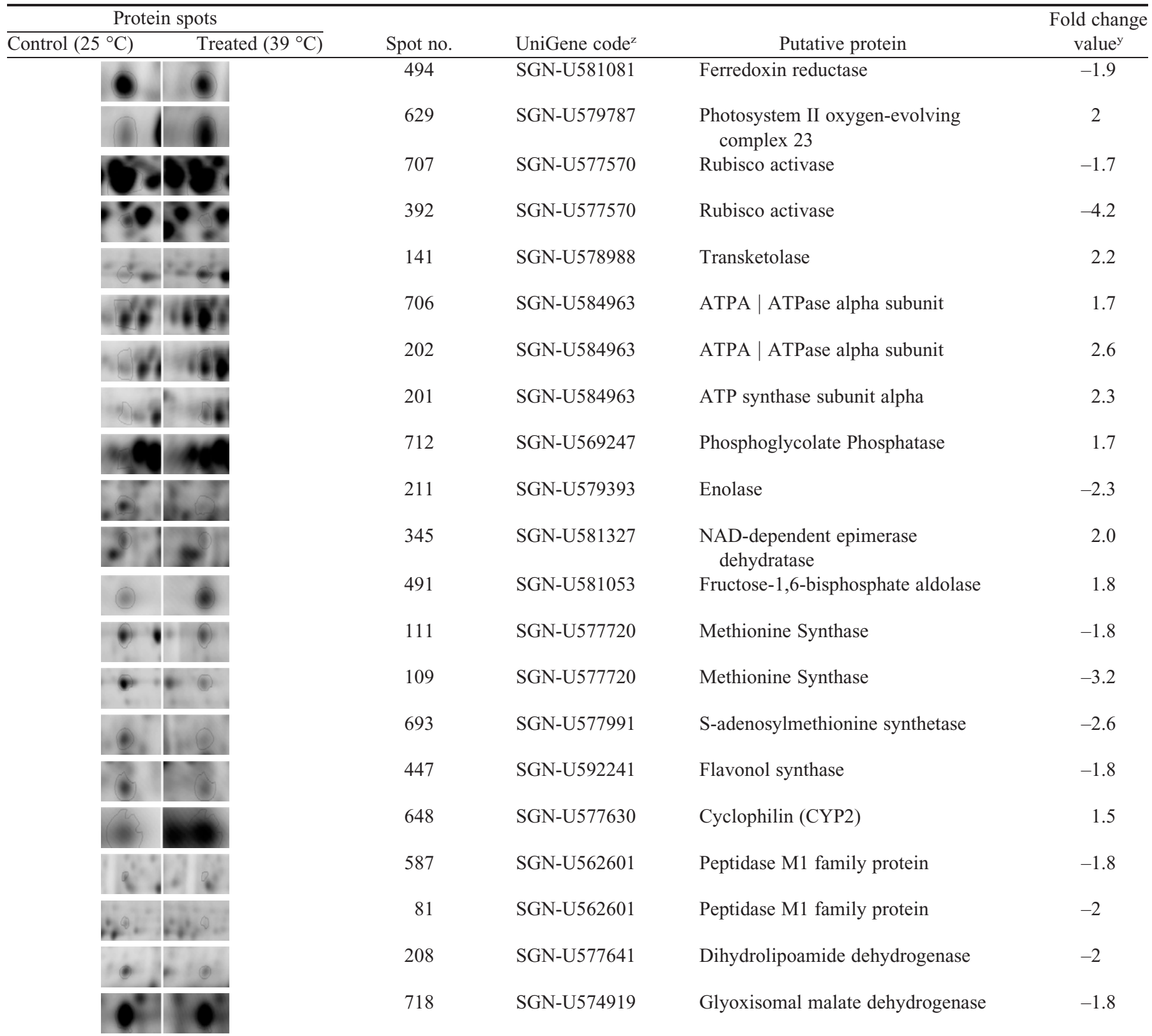

${ }^{\mathrm{z}}$ Code for each gene in the SOL Genomics Network (SGN) database.

${ }^{y}$ Fold change value is the ratio of the normalized volume of the same spot in the condition of heat-treated versus control. For example, a value of 2.0 represents a twofold increase, whereas -2.0 represents a twofold decrease from treated to control conditions. 
Table 3. Heat-induced and heat-suppressed proteins in cherry tomato LA1310.

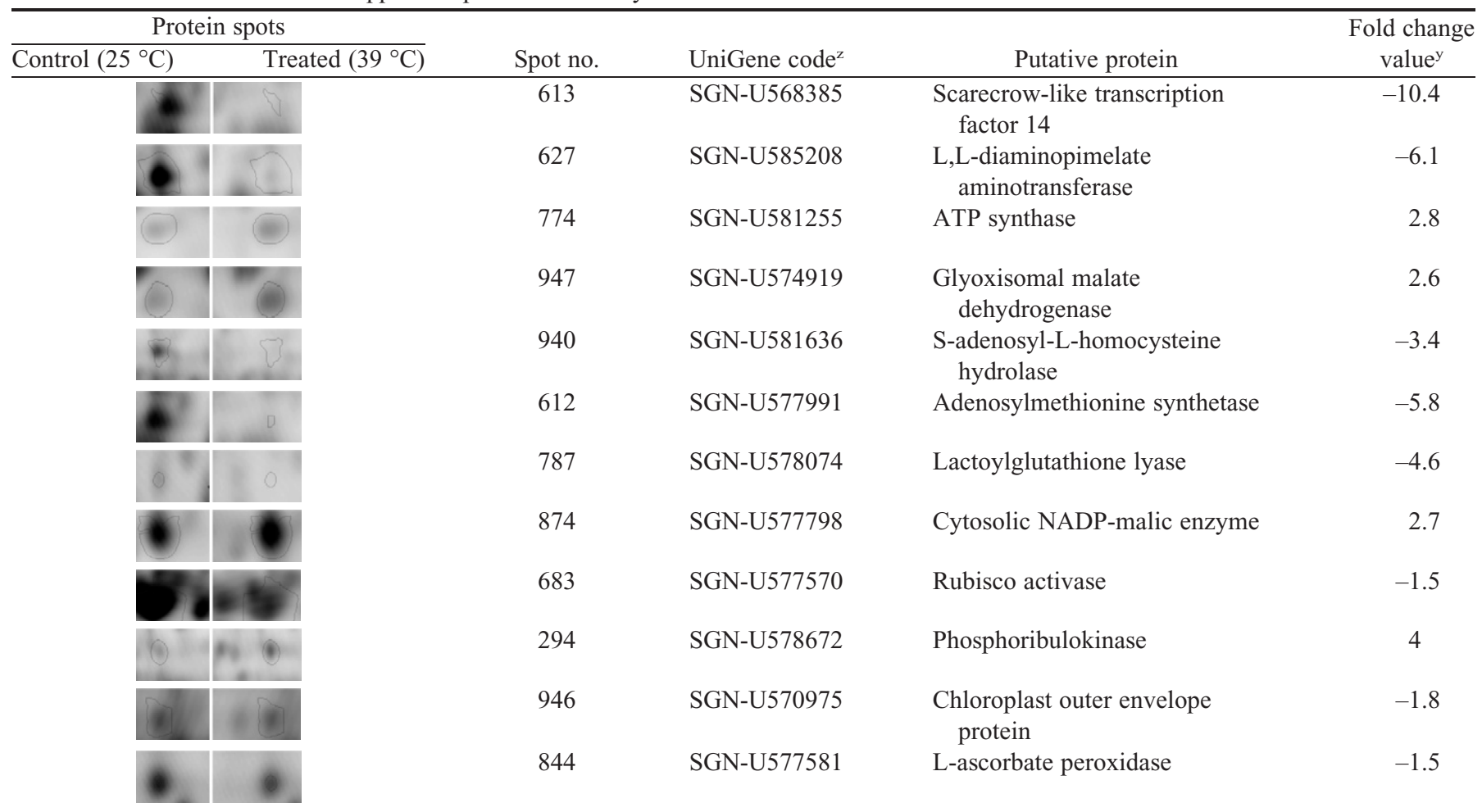

${ }^{\mathrm{z}}$ Code for each gene in the SOL Genomics Network (SGN) database.

${ }^{y}$ Fold change value is the ratio of the normalized volume of the same spot in the condition of heat-treated versus control. For example, a value of 2.0 represents a twofold increase, whereas -2.0 represents a twofold decrease from treated to control conditions.

(spot 844: -1.5 -fold), detoxification protein lactoylglutathione lyase (spot 787: -4.6-fold), and Rubisco activase (spot 683: -1.5 -fold) for carbon fixation. The heat-induced proteins were glyoxisomal malate dehydrogenase (spot 947: 2.6-fold) in glyoxalate shunt, phosphoribulokinase (spot 294: fourfold) in the Calvin cycle, and an ATP synthase (spot 774: 2.8-fold).

\section{Discussion}

Based on the heat-induced proteomes, proteins that exhibited changes in expression level following the same or contrasting patterns in two or three tomato cultivars were identified. Rubisco activase and S-adenosyl-L-homocysteine hydrolase were suppressed by heat stress in all three cultivars. Rubisco activase is a chaperon protein that modulates the activity of Rubisco (Portis, 2003; Portis et al., 2008; Spreitzer and Salvucci, 2002). Thermotolerance or heat liability of Rubisco activase is considered to play key roles in heat tolerance or susceptibility of a plant species (Kurek et al., 2007; Long and Ort, 2010; Salvucci et al., 2001). The endogenous level of Rubisco activase is an important determinant of plant productivity under heat stress conditions (Ristic et al., 2009). The Rubisco activase is present in two isoforms of 41 to $43 \mathrm{kDa}$ and 45 to $46 \mathrm{kDa}$ that arise from one alternatively spliced transcript. The larger isoform may play an important role in photosynthetic acclimation to moderate heat stress in vivo, whereas the smaller isoform plays a major role in maintaining Rubisco initial activity under normal conditions (Wang et al., 2010). In heat-treated tomato leaves, both the large and small isoforms were suppressed by heat treatment. It is therefore necessary to continue testing other heat-tolerant cultivars or wild relatives to identify heat stable Rubisco activase to increase heat tolerance of tomato cultivars.

S-adenosyl-L-homocysteine (SAM) has key functions as a primary methyl group donor and as a precursor for metabolites such as ethylene, polyamines, and osmoprotectants (Amir et al., 2002). Adenosyl L-homocysteinase is a key enzyme for the regeneration of SAM through the activated methyl cycle. Expression of the protein was suppressed by heat treatment in all three tomato cultivars; however, it was induced by salt and aluminum stresses (Krill et al., 2010; Narita et al., 2004; Zhou et al., 2009a, 2009b). These results suggest that adenosyl L-homocysteinase could play different roles in tolerance mechanisms to different stress factors.

In tomato, the major NADP-ME is a cytosolic protein and is found in developing fruit, leaves, roots, and stems (Knee et al., 1996). Overexpression of cytosolic NADP-ME increased plant defense against salt stress (Cheng and Long, 2007). The NADPME protein was induced in both 'Walter' LA3465 and cherry tomato LA1310. This suggests that NADP-ME could have an important function in stress tolerance for tomato plants. In addition, several proteins were suppressed in 'Walter' LA3465 and 'Edkawi' LA2711, but not in cherry tomato LA1310. These proteins included FNR, which is responsible for the reduction of $\mathrm{NADP}^{+}$in the PSI complex (Hurley et al., 2002) and geranylgeranyl reductase, which affects the accumulation of geranylgeranylated chlorophyll $a$ and hence the stability of photosynthetic pigment-protein complexes (Shpilyova et al., 2004; Tanaka et al., 1999). Methionine synthase was also suppressed in 'Walter' LA 3465 and 'Edkawi' LA2711. Methionine synthase is a key enzyme for the synthesis of the 
aspartate-derived methionine (Met). Met is used at multiple levels in cellular metabolism: as a protein constituent, in the initiation of mRNA translation, and as a regulatory molecule in the form of SAM (Hesse et al., 2004). In addition, the glyoxisomal malate dehydrogenase in the glycoxylate shunt was also suppressed in 'Water' LA3465 and 'Edkawi' LA2711, but it was induced in cherry tomato LA1310.

Heat stress induces production of reactive oxygen species (ROS) such as superoxide radicals, hydrogen peroxide, and hydroxyl radicals. Excess production of ROS causes oxidative damage to cellular components. In tomato, both gene expression and enzymatic activity of SOD are induced by drought and heat stresses (Panchuk et al., 2002; Perl-Treves and Galun, 1991). For tomato, SOD was induced only in 'Walter' LA3465, whereas ascorbate peroxidase (also an antioxidant enzyme) was suppressed in cherry tomato LA1310. There was no change in both enzymes in 'Edkawi' LA2711. Cyclophilins are the major group of drought- and heat-induced stress proteins (Sharma and Kaur, 2009). The cyclophilin (CYP2) chaperon protein was induced only in 'Edkawi' LA2711 but not in 'Walter' LA3465 or in cherry tomato LA1310. Tomato 'Walter' LA3465 and 'Edkawi' LA2711 are domesticated tomato forms, and cherry tomato LA1310 most likely is an ancestor of cultivated tomato (Peralta and Spooner, 2007; Ranc et al., 2008). These results suggest that the impact of these proteins on heat tolerance could be affected by the genetic background of tomato cultivars.

Heat stress-induced depletion of starch in leaves resulting from inhibition of starch formation is more pronounced in sensitive cultivars than in tolerant tomato cultivars (Dinar et al., 1983). In addition to the Calvin cycle, transketolase (TK) also participates in the oxidative pentose phosphate pathway to produces erythrose-4-phosphate. One of its substrates, fructose6-phosphate, is also the beginning point for starch synthesis, and one of its products, erythrose-4-phosphate, inhibits phosphoglucose isomerase, which catalyzes the first reaction leading to starch biosynthesis (Henkes et al., 2001). Decreased TK activity hence alters photosynthate allocation in favor of starch biosynthesis (Weber, 2007). Suppression of the TK enzyme could be used by the heat-tolerant cultivar Walter LA3465 to maintain starch concentration in leaf tissues.

In summary, heat stress suppressed the accumulation of Rubisco activase and S-adenosyl-L-homocysteine hydrolase in all tomato cultivars. Several enzymes in the glyoxylate shunt, photosynthesis, cell defense, and carbohydrate metabolism pathways were differentially expressed. This research provided the basic information needed to formulate the molecular regulatory mechanism for heat tolerance in tomato.

\section{Literature Cited}

Alban, A., S.O. David, L. Bjorkesten, C. Andersson, E. Sloge, S. Lewis, and I. Currie. 2003. A novel experimental design for comparative twodimensional gel analysis: Two-dimensional difference gel electrophoresis incorporating a pooled internal standard. Proteomics 3:36-44.

Amir, R., Y. Hacham, and G. Galili. 2002. Cystathionine gammasynthase and threonine synthase operate in concert to regulate carbon flow towards methionine in plants. Trends Plant Sci. 7:153-157.

Berry, J.A. and O. Björkman. 1980. Photosynthetic response and adaptation to temperature in higher plants. Annu. Rev. Plant Physiol. 31:491-543.

Berry, S.Z. and M. Rafique-Ud-Din. 1988. Effect of high temperature on fruit set in tomato cultivars and selected germplasm. HortScience 23:606-608.
Bohnert, H.J., Q. Gong, P. Li, and S. Ma. 2006. Unraveling abiotic stress tolerance mechanisms - Getting genomics going. Curr. Opin. Plant Biol. 9:180-188.

Camejo, D., A. Jiménez, J.J. Alarcón, W. Torres, J.M. Gómez, and F. Sevilla. 2006. Changes in photosynthetic parameters and antioxidant activities following heat-shock treatment in tomato plants. Funct. Plant Biol. 33:177-187.

Camejo, D., P. Rodríguez, M.A. Morales, J.M. Dell'amico, A. Torrecillas, and J.J. Alarcón. 2005. High temperature effects on photosynthetic activity of two tomato cultivars with different heat susceptibility. J. Plant Physiol. 162:281-289.

Chan-Schaminet, K.Y., S.K. Baniwal, D. Bublak, L. Nover, and K.D. Scharf. 2009. Specific interaction between tomato HsfA1 and HsfA2 creates hetero-oligomeric superactivator complexes for synergistic activation of heat stress gene expression. J. Biol. Chem. 284:2084820857.

Cheng, Y. and M. Long. 2007. A cytosolic NADP-malic enzyme gene from rice (Oryza sativa L.) confers salt tolerance in transgenic Arabidopsis. Biotechnol. Lett. 29:1129-1134.

Dinar, M., J. Rudich, and E. Zamski. 1983. Effects of heat stress on carbon transport from tomato leaves. Ann. Bot. (Lond.) 51:97103.

Foolad, M.R. 2005. Breeding for abiotic stress tolerances in tomato, p. 613-684. In: Ashraf, M. and P.J.C. Harris (eds.). Abiotic stresses: Plant resistance through breeding and molecular approaches. Haworth Press, New York, NY.

Frank, G., E. Pressman, R. Ophir, L. Althan, R. Shaked, M. Freedman, S. Shen, and N.J. Firon. 2009. Transcriptional profiling of maturing tomato (Solanum lycopersicum L.) microspores reveals the involvement of heat shock proteins, ROS scavengers, hormones, and sugars in the heat stress response. Expt. Bot 60:3891-3908.

Henkes, S., U. Sonnewald, R. Badur, R. Flachmann, and M. Stitt. 2001. A small decrease of plastid transketolase activity in antisense tobacco transformants has dramatic effects on photosynthesis and phenylpropanoid metabolism. Plant Cell 13:535-552.

Hesse, H., O. Kreft, S. Maimann, M. Zeh, and R. Hoefgen. 2004. Current understanding of the regulation of methionine biosynthesis in plants. J. Expt. Bot. 55:1799-1808.

Hurley, J.K., R. Morales, M. Martínez-Júlvez, T.B. Brodie, M. Medina, C. Gómez-Moreno, and G. Tollin. 2002. Structure-function relationships in Anabaena ferredoxin/ferredoxin-NADP ${ }^{+}$reductase electron transfer: Insights from site-directed mutagenesis, transient absorption spectroscopy and X-ray crystallography. Biochim. Biophys. Acta 1554:5-21.

Ismail, A.M. and A.E. Hall. 1999. Reproductive-stage heat tolerance, leaf membrane thermostability and plant morphology in cowpea. Crop Sci. 39:1762-1768.

Karim, M.A., Y. Fracheboud, and P. Stamp. 1999. Photosynthetic activity of developing leaves is less affected by heat stress than that of developed leaves. Physiol. Plant. 105:685-693.

Karp, N.A. and K.S. Lilley. 2007. Design and analysis issues in quantitative proteomics studies. Proteomics 7(suppl):42-50.

Karp, N.A., P.S. McCormick, M.R. Russell, and K.S. Lilley. 2007. Experimental and statistical considerations to avoid false conclusions in proteomics studies using differential in-gel electrophoresis. Mol. Cell. Proteomics 6:1354-1364.

Knee, M., F.L. Finger, and L.M. Lagrimini. 1996. Evidence for a cytosolic NADP-malic enzyme in tomato. Photochemistry 42:11-16. Krill, A.M., M. Kirst, L.V. Kochian, E.S. Buckler, and O.A. Hoekenga. 2010. Association and linkage analysis of aluminum tolerance genes in maize. PLoS One 5:e9958.

Kurek, I., T.K. Chang, S.M. Bertain, A. Madrigal, L. Liu, M.W. Lassner, and G. Zhu. 2007. Enhanced thermostability of Arabidopsis Rubisco activase improves photosynthesis and growth rates under moderate heat stress. Plant Cell 19:3230-3241.

Link, V., A. Sinha, P. Vashista, M. Hofmann, R. Proels, R. Ehness, and T. Roitsch. 2002. A heat-activated MAP kinase in tomato: A possible regulator of the heat stress response. FEBS Lett. 531:179-183. 
Long, S.P. and D.R. Ort. 2010. More than taking the heat: Crops and global change. Curr. Opin. Plant Biol. 13:1-8.

Murakami, Y., M. Tsuyama, Y. Kobayashi, and H. Kodama. 2000. Trienoic fatty acids and plant tolerance of high temperature. Science 287:476-479.

Narita, Y., H. Taguchi, T. Nakamura, A. Ueda, W. Shi, and T. Takabe. 2004. Characterization of the salt-inducible methionine synthase from barley leaves. Plant Sci. 167:1009-1016.

Panchuk, I.I., R.A. Volkov, and F. Schöffl. 2002. Heat stress- and heat shock transcription factor-dependent expression and activity of ascorbate peroxidase in Arabidopsis. J. Plant Physiol. 129:838-853.

Peralta, I.E. and D.M. Spooner. 2007. History, origin and early cultivation of tomato (Solanaceae), p. 1-27. In: Razdan, M.K. and A.K. Mattoo (eds.). Genetic improvement of Solanaceous crops, Vol. 2. Tomato. Science Publishers, Enfield, NH.

Perl-Treves, R. and E. Galun. 1991. The tomato $\mathrm{Cu}, \mathrm{Zn}$ peroxide dismutase genes are developmentally regulated and respond to light and stress. Plant Mol. Biol. 17:745-760.

Port, M., J. Tripp, D. Zielinski, C. Weber, D. Heerklotz, S. Winkelhaus, D. Bublak, and K.D. Scharf. 2004. Role of Hsp17.4-CII as coregulator and cytoplasmic retention factor of tomato heat stress transcription factor HsfA2. J. Plant Physiol. 135:1457-1470.

Portis, A. 2003. RUBISCO activase: RUBISCO's catalytic chaperone. Photosynth. Res. 75:11-27.

Portis, J.R., C. Li, D. Wang, and M.E. Salvucci. 2008. Regulation of Rubisco activase and its interaction with Rubisco. J. Expt. Bot. 59: 1597-1604.

Ranc, N., S. Muños, S. Santoni, and M. Causse. 2008. A clarified position for Solanum lycopersicum var. cerasiforme in the evolutionary history of tomatoes (solanaceae). BMC Plant Biol. 8:130.

Ristic, Z., I. Momcilovic, U. Bukovnik, P.V. Prasad, J. Fu, B.P. Deridder, T.E. Elthon, and N. Mladenov. 2009. Rubisco activase and wheat productivity under heat-stress conditions. J. Expt. Bot. 60:4003-4014.

Saeed, A., K. Hayat, A.A. Khan, and S. Iqbal. 2007. Heat tolerance studies in tomato (Lycopersicon esculentum Mill.). Intl. J. Agr. Biol. 9:649-652.

Salvucci, M.E., K.W. Osteryoung, S.J. Crafts-Brandner, and E. Vierling. 2001. Exceptional sensitivity of Rubisco activase to thermal denaturation in vitro and in vivo. J. Plant Physiol. 127: 1053-1064.

Scharf, K.D., H. Heider, I. Höhfeld, R. Lyck, E. Schmidt, and L. Nover. 1998. The tomato Hsf system: HsfA2 needs interaction with HsfA1 for efficient nuclear import and may be localized in cytoplasmic heat stress granules. Mol. Cell. Biol. 18:2240-2251.
Schultheiss, J., O. Kunert, U. Gase, K.D. Scharf, L. Nover, and H. Rüterjans. 1996. Solution structure of the DNA-binding domain of the tomato heat-stress transcription factor HSF24. Eur. J. Biochem. 236:911-921.

Sharma, A.D. and P. Kaur. 2009. Combined effect of drought stress and heat shock on cyclophilin protein expression in Triticum aestivum. Gen. Appl. Plant Physiol. 35:88-92.

Shpilyova, A.V., V.V. Zinchenkoa, S.V. Shestakova, B. Grimmb, and H. Lokstein. 2004. Inactivation of the geranylgeranyl reductase (ChlP) gene in the cyanobacterium Synechocystis sp. PCC 6803. Biochimica et Biophysica Acta Bioenergetics 1706:195-203.

Spreitzer, R.J. and M.E. Salvucci. 2002. RUBISCO: Structure, regulatory interactions, and possibilities for a better enzyme. Annu. Rev. Plant Biol. 53:449-475.

Swift, C. 2008. Heat-resistant vegetable varieties for Florida. Tomato and other seed and plant varieties to grow in Florida. 3 Jan. 2011. $<\mathrm{http}$ //www.suite101.com/content/heatresistant-vegetable-varietiesfor-florida-a116133>.

Tanaka, R., U. Oster, E. Kruse, W. Rudiger, and B. Grimm. 1999. Reduced activity of geranylgeranyl reductase leads to loss of chlorophyll and tocopherol and to partially geranylgeranylated chlorophyll in transgenic tobacco plants expressing antisense RNA for geranylgeranyl reductase. J. Plant Physiol. 120:695-704.

U.S. Global Change Research Program. 2008. Our changing planet. 3 Jan. 2011. <http://www.usgcrp.gov/usgerp/Library/ocp2008/ocp2008. pdf $>$.

Wahid, A., S. Gelani, M. Ashraf, and M.R. Foolad. 2007. Heat tolerance in plants: An overview. Environ. Exp. Bot. 61A:199-223.

Wang, D., X.F. Li, Z.J. Zhou, X.P. Feng, W.J. Yang, and D.A. Jiang. 2010. Two Rubisco activase isoforms may play different roles in photosynthetic heat acclimation in the rice plant. Physiol. Plant. 139: $55-67$.

Weber, A.P.M. 2007. Synthesis, export and partitioning of the end products of photosynthesis, p. 273-292. In: Wise, R.R. and J.K. Hoober (eds.). The structure and function of plastids. Springer, Dordrecht, The Netherlands.

Zhang, S., C.K. Van Pelt, and J.D. Henion. 2003. Automated chipbased nano electrospray-mass spectroscopy for rapid identification of proteins separated by two-dimensional electrophoresis. Electrophoresis 24:3620-3632.

Zhou, S., R. Sauve, T. Fish, and T.W. Thannhauser. 2009a. Salt induced protein in tomato leaves. J. Amer. Soc. Hort. Sci. 134:289-294.

Zhou, S., R. Sauve, and T.W. Thannhauser. 2009b. Proteome changes induced by aluminum stress in tomato roots. J. Expt. Bot. 60:18491857. 